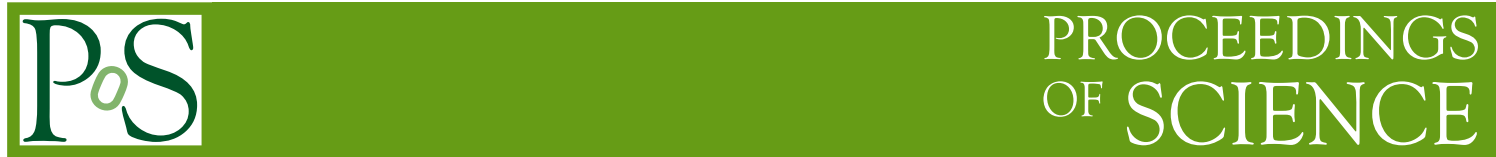

\title{
Top quark physics at DØ
}

\section{Peter Renkel for the D $\varnothing$ collaboration*}

Southern Methodist Univeristy, USA

E-mail: renkelefnal.gov

We give an overview of measurements by the $D \emptyset$ experiment of properties of the top quark and searches for new physics in the top sector. The data are for integrated $p \bar{p}$ luminosities of up to 5.3 $\mathrm{fb}^{-1}$ at a center-of-mass energy of $1.96 \mathrm{TeV}$. We highlight measurements of the top-antitop quarkpair production cross section, top quark width couplings, and top-antitop quark spin correlations, the top quark mass, and searches for the fourth generation particles.

The Xth Nicola Cabibbo International Conference on Heavy Quarks and Leptons,

October 11-15, 2010

Frascati (Rome) Italy

\footnotetext{
* Speaker.
} 


\section{Top quark production}

After the top $(t)$ quark was discovered in 1995 [1], emphasis quickly turned to detailed studies of its properties, including measuring its production cross section, its mass across all reconstructable final states, and other properties, such as spin, charge and couplings.

The $t \bar{t}$ production cross section can be calculated in the standard model (SM) with a precision of 6-8\%. Any deviation of its measured value from the SM prediction could signal the presence of new physics in production or $t \bar{t}$ decays. In the SM, top quarks in $\sim 100 \%$ cases decay into a $b$ quark and a $W$ boson. If the decay into a charged Higgs and a $b$ quark $\left(t \rightarrow H^{ \pm} b\right)$ is allowed, the contributions of $t$ decays to to different final states can change, indicating a new physics. It is, therefore, important to measure the $t \bar{t}$ production cross section with precision in all channels.

The $\mathrm{D} \emptyset$ collaboration recently measured the $t \bar{t}$ production cross section at $p \bar{p}$ collisions at the Tevatron in $l+$ jets final states [2], where one $W$ boson from the top quark decays into a light lepton (excluding $\tau$ ) and a neutrino, and another $W$ boson decays purely hadronically. Measuring the cross section in this channel is challenging because of the significant $W+$ jets background, where the $W$ boson decays leptonically. To reduce this background, we tag $b$ jets, that is, jets coming from $b$ quarks by reconstructing their secondary vertices and requiring significant displacement of such vertices from the primary $p \bar{p}$. We also reduce this background by utilizing the kinematics of the $t \bar{t}$ events, such as the invariant masses of combinations of different objects in the $t \bar{t}$ events or the scalar sums of their momenta. We extract the $t \bar{t}$ production cross section: $\sigma_{t \bar{t}}^{l+j e t s}=7.70_{-0.70}^{+0.79}$ $($ stat + syst + lumi $) \mathrm{pb}$ in the $l+$ jets final states.

The $t \bar{t}$ production cross section is also measured in dilepton final states, where both $W$ bosons decay leptonically [3]. As in case of the $l+$ jets measurement, we use the topology of this final state to discriminate signal against the background.

Figure 1 shows a comparison of data to Monte Carlo (MC) generated events for several variables in lepton + jets and dilepton channels: (a) discriminating output, (b) jet multiplicity for the lepton + jets channel, and (c) missing transverse mass, (d) the scalar sum of the transverse momenta of the leading lepton and jets $H_{T}$ for the dilepton analyses distributions. The data and MC agree in all distributions. We extract a $t \bar{t}$ production cross section of $\sigma_{t}^{\text {dilepton }}=8.4+0.5$ (stat) ${ }_{-0.8}^{+0.9}$ (syst) ${ }_{-0.6}^{+0.7}$ (lumi) pb.

The DØ Collaboration also measured the dependence of the $t \bar{t}$ differential cross section on the top quark transverse momentum $p_{T}$ [4]. This is important for checking consistency with the next to leading order (NLO) generators. Figure 2 shows the $p_{T}$ dependence in $l+$ jets events. It is clear from the comparison that the data agrees better with NLO generators generators such as NLO pQCD and MC@NLO than with LO generators PYTHIA and ALPGEN.

After discovery of single top quark production [5], the emphasis moved to analyzing its $s$ channel and $t$-channels separately, and measuring their separate contributions to the production cross sections [6]. In this analysis, we require exactly one isolated lepton and two to four jets, and apply three independent multivariate techniques: Boosted Decision Trees, Bayesian Neural Networks, and the Matrix Element method. Figure 3 (a) shows the posterior probability density for $s$ and $t$-channels. We measure cross sections of $3.14_{-0.80}^{+0.94} \mathrm{pb}$ for the t-channel and $1.05 \pm 0.81 \mathrm{GeV}$ for the $s$-channel. 


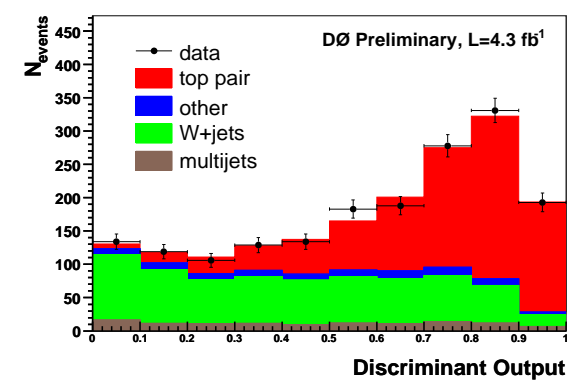

(a)

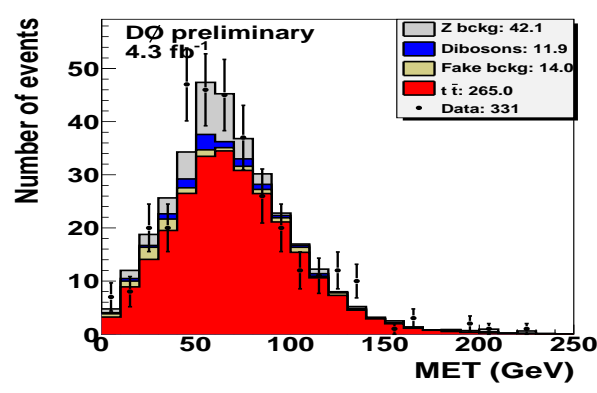

(c)

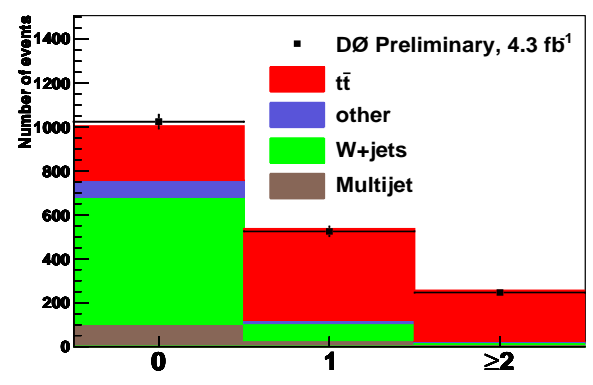

(b)

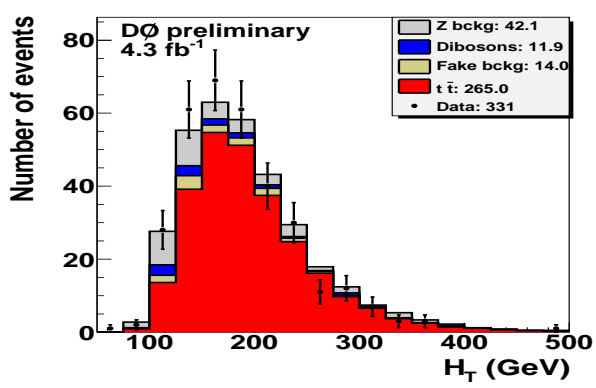

(d)

Figure 1: Comparison of distribution for data and simulations in (a) discriminant output and (b) jet multiplicity for the lepton + jets analysis, and (c) missing energy , (d) $H_{T}$ for the dilepton analysis distributions for data and Monte Carlo

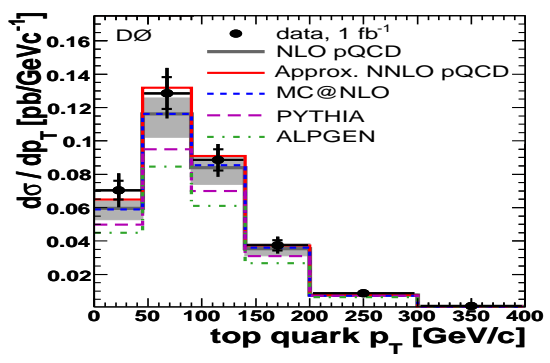

Figure 2: Differential cross section as a function of the $p_{T}$ of the top quark in $l+$ jets events

The single top quark final state is sensitive to several models of physics beyond the SM, especially those in which flavor-changing neutral current (FCNC) couplings between a gluon, a top quark and up or charm quarks is large. Examples include models with multiple Higgs doublets, those with new dynamic interactions of the top quark, or models in which the top quark is a composite object or has a soliton structure. The $\mathrm{D} \emptyset$ collaboration has performed a search for FCNC in quark-gluon couplings in a sample of single top quark final states [7]. We find consistency between background expectation and observed data, and set limits on FCNC gluon couplings of the top quark to up quarks (tgu) and charm quarks $(\operatorname{tgc})$. Figure 3 shows the limits on the corresponding cross sections, which sets limits on decay branching fractions of the top quark of $B(t \rightarrow g u)<2.4$ 


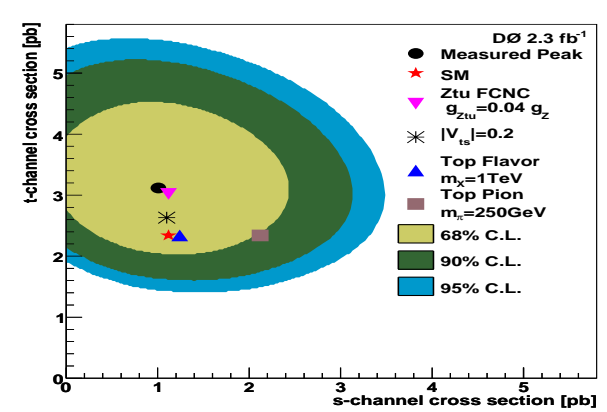

(a)

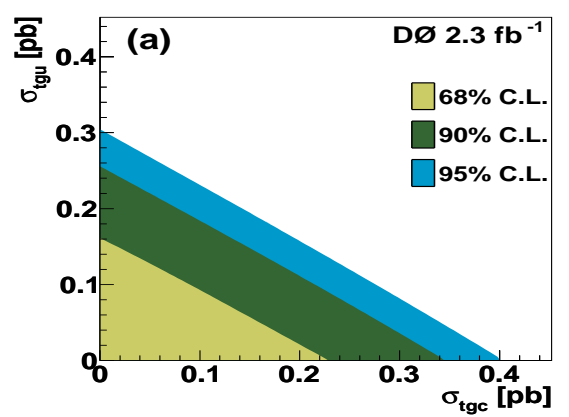

(b)

Figure 3: (a) 2-dimensional histogram for the t-channel and s-channel and (b) Bayesian limits on the posterior probability as a function of the $\sigma_{t g u}$ and $\sigma_{t g c}$ contribution to single top

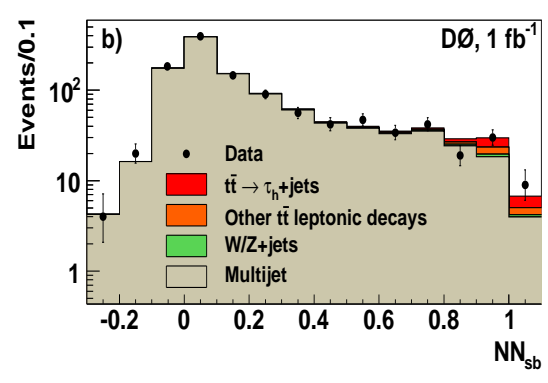

(a)

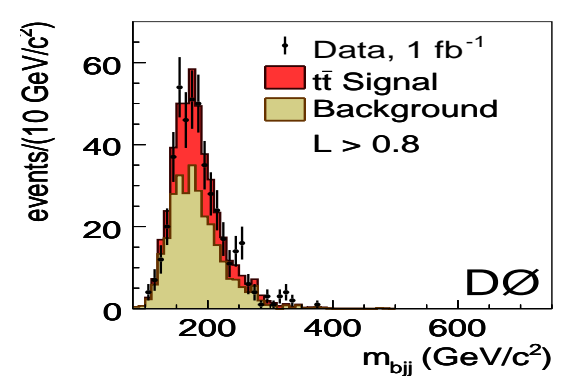

(b)

Figure 4: Distribution in (a) Neural network for the $\tau+$ jets channel and (b) the invariant mass of two jets and a $b$ tagged jet in the all jets channel

and $B(t \rightarrow g c)<3.3$.

We also measure the $t \bar{t}$ production cross section in the $\tau+$ jets channel, where we have at least one isolated $\tau$ hadron in the final state [8], as well as $\sigma_{t \bar{t}}$ in all jets final state [9]. Figure 4 shows comparison of characteristic distributions in each case. We measure cross sections of $\sigma_{t \bar{t}}=6.9 \pm 1.2$ (stat) ${ }_{-0.7}^{+0.8}$ (syst) from the $\tau+$ jets channel and $\sigma_{t \bar{t}}=6.9 \pm 2.0 \mathrm{pb}$ for the all jets channel.

\section{Top quark properties}

Within the standard model, a precise measurement of the top quark mass $\left(m_{t}\right)$ and $W$ boson mass $\left(M_{W}\right)$ can be used to constrain the Higgs boson mass $\left(M_{H}\right)$. We measure $m_{t}$ in the $l+$ jets channel by using the Matrix Element approach [10].

As in case of the cross section measurement, we define our sample by requiring at least one isolated lepton and jets. We calibrate the jet energy scale (JES) in-situ by requiring the invariant mass of the two light jets to be equal to the mass of the $W$ boson. Figure 5 shows the result from a fit to a likelihood in JES and $m_{t}$, and a plot of the calibrated projection on the $m_{t}$ axis.

We obtain $m_{t}=173.7 \pm 0.8$ (stat) \pm 1.6 (syst). 


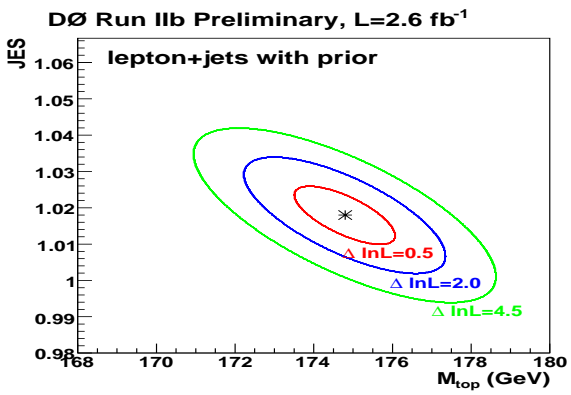

(a)

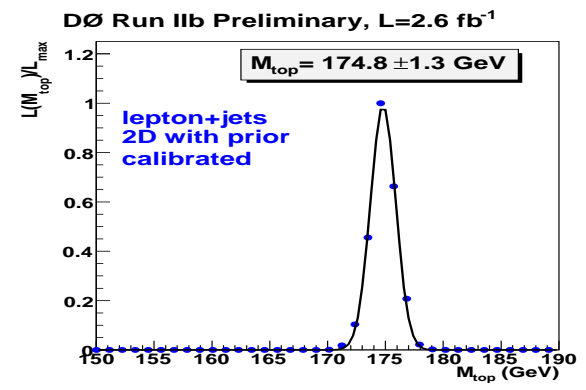

(b)

Figure 5: Studies of the mass of the top quark in $l+$ jets channel: (a) the result from a fit to a likelihood in JES and $m_{t}$, and (b) a plot of the calibrated projection on the $m_{t}$ axis

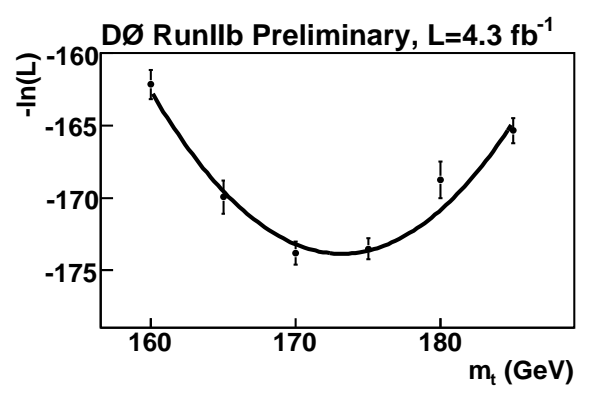

(a)

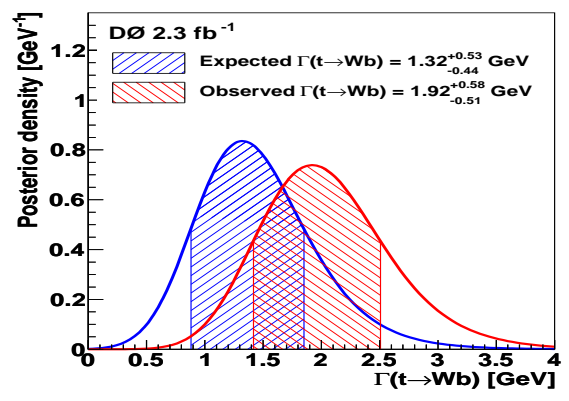

(b)

Figure 6: (a) Negative log-likelihood function for data before calibration, as a function of $m_{t}$. (b) Probability density for the expected and measured partial width $\Gamma(t \rightarrow W b)$

We also perform a measurement of the top quark mass in the $e \mu$ dilepton channel [11], where we require one electron, one muon, at least two jets, and significant $H_{T}$. The four-vectors of neutrinos cannot be reconstructed, and we therefore integrate over the unknown parameters (pseudorapidities). We use a template method for the mass reconstruction.

The negative log-likelihood as a function of $m_{t}$ for data before calibration is shown on Fig. 6 (a). We obtain a mass of $m_{t}^{\text {dilepton }}=173.3 \pm 2.4$ (stat) \pm 2.1 (syst)

We also measure other properties of the top quark, one example being its width. We extract the total width of the top quark [12] from the partial decay width $\Gamma(t \rightarrow W b)$ measured using the $t$-channel cross section for single top quark production and from the branching fraction $B(t \rightarrow W b)$ measured in $t \bar{t}$ events. The result is $\Gamma_{t}=1.99_{-0.55}^{+0.69} \mathrm{GeV}$, which translates to a top-quark lifetime of $\tau_{t}=\left(3.3_{-0.9}^{+1.3}\right) \times 10^{-24} \mathrm{~s}$. The probability density for the expected and measured partial width $\Gamma(t \rightarrow W b)$ is given on Fig.6 (b).

The SM predicts that the top quark decays before its spin projection can change. This is in contrast with the lighter quarks, which are depolarized through QCD interactions well before they fragment. The spin of the top quark is therefore reflected in its decay products. If top quarks decay as predicted in the SM, then the charged lepton from the $W$ decay has a spin analyzing power of 1 at leading order in QCD. Consistently dilepton final states have the highest sensitivity to the correlation between the spins of pair-produced top and antitop quarks. We measure the strength of 


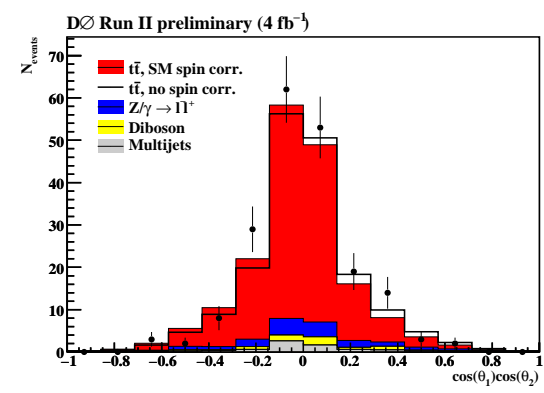

(a)

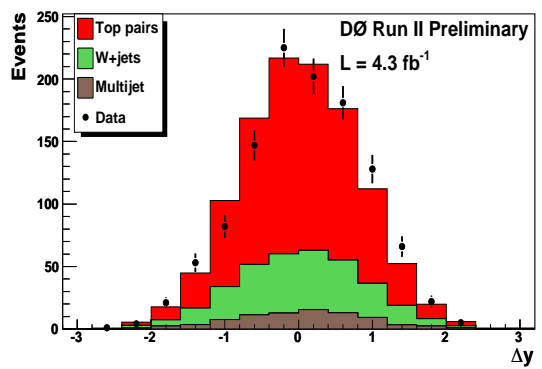

(b)

Figure 7: (a) The distribution of the product of cosine of angles between a lepton(antilepton) in the top(antitop) rest frame and the beam direction for the full dilepton event sample. (b) The sum of MC $t \bar{t}$ signal including the expected NLO QCD spin correlation $(\mathrm{C}=0.777)$ (red) and multijet (gray), diboson (yellow) and Drell-Yan (blue) production is compared to data. The black line shows the prediction without $t \bar{t}$ spin correlation $(\mathrm{C}=0)$. The comparison of data and signal plus background for the rapidity difference between $t$ and $\bar{t}$ events in $t \bar{t}$ final states

spin correlations (C) [13]. Fig. 7 (a) shows the distribution for all dilepton events. The correlation strength is found to be $\mathrm{C}=0.17 \pm 0.65 \pm 0.53$ (stat + syst), in agreement of $2 \sigma$ with the $\mathrm{SM}$ prediction of $\mathrm{C}=0.777$.

At the Tevatron, the color-charge asymmetry is observable as a forward-backward asymmetry in the angular distribution of the top or antitop quarks in $t \bar{t}$ events. At NLO in QCD, asymmetries of five to ten percent appear. Some extensions of the standard model, such as $Z \rightarrow t \bar{t}$ decays and warped extra dimensions, predict higher asymmetries. Some, such as axigluons, predict lower asymmetries. We define forward and backward events by the sign of their difference in pseudorapidity and then define the forward-backward asymmetry $\left(A_{f b}\right)$ to be the difference of the number of forward events and the number of backward events divided by the total number of events [14]. Figure 7 (a) shows the comparison of data and signal plus background for the rapidity difference. We observe an asymmetry of $A_{f b}=(8 \pm 4$ (stat) \pm 1 (syst) $) \%$. This is $2 \sigma$ from the SM prediction.

The CPT theorem, which underlines any local Lorentz-invariant quantum field theory, requires that the mass of a particle and that of its antiparticle be identical. We check this requirement at the Tevatron by comparing the masses of top and antitop quarks [15]. We measure the top quark mass using the ME method. Figure 8 shows fits to the likelihoods as a function of of top and antitop masses for the $e+$ jets and $\mu+$ jets channels respectively. The measured mass difference of $3.8 \pm 3.7$ $\mathrm{GeV}$ is consistent with the equality of top and antitop masses at the level of $\sim 2 \%$.

\section{Searches}

Measurements at LEP of the partial width of the $\mathrm{Z}$ boson to invisible final states exclude the existence of a fourth neutrino with mass less than half the $\mathrm{Z}$ boson mass. However, this does not exclude a sequential fourth generation of fermions. The $D \emptyset$ collaboration has soughed a fourth generation top quark that is produced in proton-antiproton collisions together with its antiparticle [16]. We assume that such fourth generation quark always decays to a $W$ boson and a top quark. We 


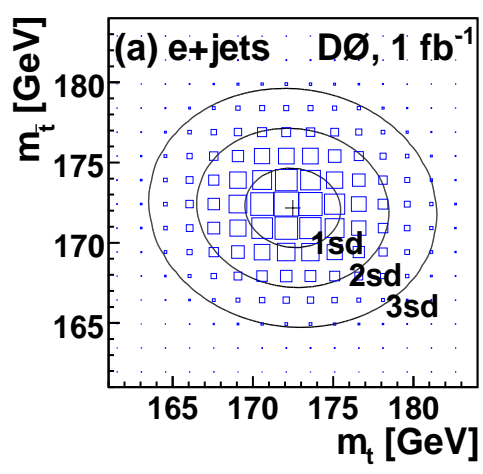

(a)

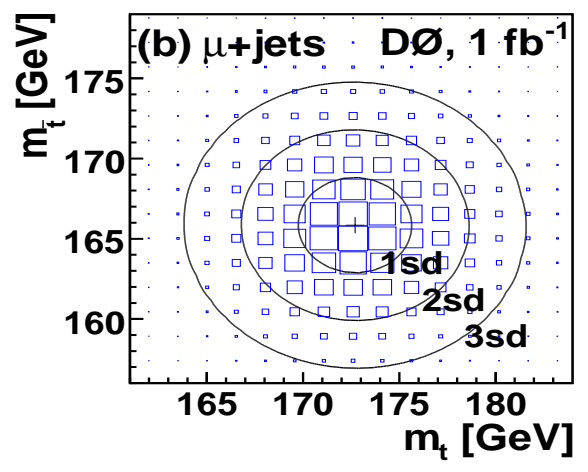

(b)

Figure 8: Measurement of $m_{t}$ vs $m_{\bar{t}}$ used to obtain the top - antitop mass difference for the (a) $e+j e t s$ and (b) $\mu+$ jets channels respectively.

set upper limits on the production cross section of such quarks which are used to exclude at $95 \%$ confidence level such fourth generation quark with a mass below $296 \mathrm{GeV}$.

\section{References}

[1] D0 Collaboration, V.M. Abazov et al., Phys. Rev. Lett. 74, 2632 (1995)

[2] D0 Collaboration, D0-6037-CONF (2010)

[3] D0 Collaboration, D0-6038-CONF (2010)

[4] D0 Collaboration, V.M. Abazov et al., Physics Letters B 693 (2010) 515-521

[5] D0 Collaboration, V.M. Abazov et al., Phys. Rev. Lett. 103, 092001 (2009)

[6] D0 Collaboration, V.M. Abazov et al., Phys. Lett. B 682, 363 (2010)

[7] D0 Collaboration, V.M. Abazov et al., Phys.Lett.B693:81-87,(2010)

[8] D0 Collaboration, V.M. Abazov et al., Fermilab-PUB-10/333-E(2010)

[9] D0 Collaboration, V.M. Abazov et al., Fermilab-PUB-09/592-E(2010)

[10] D0 Collaboration, D0-5877-CONF (2010)

[11] D0 Collaboration, D0-6104-CONF (2010)

[12] D0 Collaboration, V.M. Abazov et al., Fermilab-PUB-10/394-E(2010)

[13] D0 Collaboration, D0-5950-CONF (2010)

[14] D0 Collaboration, V.M. Abazov et al.,D0-6062-CONF(2010)

[15] D0 Collaboration, V.M. Abazov et al., Phys.Rev.Lett.103.132001 (2009)

[16] D0 Collaboration, V.M. Abazov et al., D0-5892-CONF (2010) 